\title{
Turbulence modeling in the atmospheric boundary layer: a review and some recent developments
}

\author{
J. G. Bartzis \\ Department of Energy and Resources Management Engineering, \\ University of West Macedonia, Kozani, Greece
}

\begin{abstract}
Wind flow and pollutant dispersion are the main two points of interest that make the prediction of the atmospheric flow a necessity for the modern society. Atmospheric problems are an important CFD application field and have repeatedly boosted CFD development. CFD-LES methodology is more attractive to local scale problems and neutral flows. CFD-RANS methodology is the main tool used today for practical problems. The problem of turbulence closure scheme selection for CFD-RANS applications is still an open question in atmospheric flows. For local scale problems the two equation turbulence modelling approach is the dominant one and especially the standard k- $\varepsilon$ model. In the literature one can find simpler models (empirical, one equation models) or higher models as well as Reynolds stress models. Recently, the k- $\varepsilon$ model has been re-examined and a new general approach in developing two-equation turbulence models is proposed with the aim of improving their reliability and consequently their range of applicability. The results up to now are quite encouraging.
\end{abstract}

Keywords: atmospheric boundary layer, CFD modelling, $k-\zeta$ model, turbulence closure, turbulent length scale, urban canyon.

\section{Introduction}

Weather forecast and pollutant dispersion are the main two points of interest that make the prediction of the atmospheric flow a necessity for the modern society. Atmospheric problems are an important CFD application field and have repeatedly boosted CFD development. Other methods to calculate the flow still 
exist, but CFD has the advantage of becoming more accurate as computers develop and also being able to give results even in difficult cases, like complex terrain. Especially in local scale problems with high complexity, CFD is almost the only way to have reasonable results.

The applications that utilize direct numerical solution (DNS) of the NavierStokes equations are very limited in the atmosphere. They have to do more with low Reynolds number and underlying flow regimes rather than real flows.

Volume averaged Navier-Stokes are used from the so-called LES method where the only fluctuation values that are parameterised are the subgrid values (small turbulent eddies), while the large eddies are calculated explicitly. The method is more attractive to local scale and neutral flows where the methodology can be applied more easily.

On the other hand the ensemble averaged Navier-Stokes (RANS) are more commonly used. The RANS methodology is mainly used today for practical problems [1].

\section{The CFD-RANS turbulence closure problem}

The problem of turbulence closure scheme selection is still an open question in atmospheric flows. The most straightforward approach to estimate the Reynolds stresses is to generate transport conservation equations (RSM) for each one of them. The parameterisation of these equations is a relatively difficult task due to the complexity of its redistribution terms and source terms. The level 4 model of Mellor-Yamada ([2, 3]) is a classical choice for the limited number of users who want to use such models in the atmosphere today. The RSM models have been used even from the mid seventies but they were not found to produce adequately better results than the more simple ones to defend their much bigger computation time and complexity.

Concerning the turbulence closure, a more simple approach based on the concept of the eddy viscosity/diffusivity remains still the most attractive approach for practical problems today. A usual definition of the eddy viscosity $(\mathrm{K})$ is given by the relation:

$$
-\overline{u_{i}^{\prime} u_{j}^{\prime}}=K\left(\frac{\partial u_{i}}{\partial x_{j}}+\frac{\partial u_{j}}{\partial x_{i}}\right)-\frac{2}{3} k \delta_{i j} .
$$

The simplest approach is to utilize empirical relationships for K. Such a method is still used with success in mesoscale flows (e.g. [6]). For simple flows or when high accuracy is not a priority, simple empirical models are sufficient to be used. The fact that those models are being used for so many years has resulted an accumulated experience with regards to their performance. Holt and Raman in [7] give a complete review of most of these models.

The most common approach however especially for local flows is based on Prandtl's hypothesis [4] in which the $\mathrm{K}$ parameter is scaled as $K \sim U \cdot \ell$ where $U$ is the velocity scale representing turbulence 'intensity' and $\ell$ is a length 
scale directly related to the length scale of turbulence eddies. Regarding $\mathrm{U}$ estimation, the most successful hypothesis seems to be that of Prandtl's suggesting $\quad \mathrm{U} \sim k^{1 / 2}$ where $\mathrm{k}$ is the turbulent kinetic energy (TKE).

The modelling of the TKE equation is much easier than that of the Reynolds stresses equations and is considered accurate enough. Its standard form which is more or less universally accepted has as follows:

$$
\begin{gathered}
\frac{\partial \rho k}{\partial t}+\frac{\partial \rho u_{j} k}{\partial x_{j}}=\frac{\partial}{\partial x_{j}}\left(\rho \frac{K}{\sigma_{k}} \frac{\partial k}{\partial x_{j}}\right)+\rho S_{k} \\
S_{k}=-\overline{u_{i}^{\prime} u_{j}^{\prime}} \frac{\partial u_{i}}{\partial x_{j}}+\overline{\rho^{\prime} w^{\prime}} g / \rho-\varepsilon
\end{gathered}
$$

The $\varepsilon$ parameter is the turbulent energy dissipation.

The parametrization of the length scale (and $\varepsilon$ ) is given either empirically (one equation models) or through a transport equation for an additional turbulent parameter (two equation models).

Many mesoscale models for practical applications use one-equation models. They are simple and accurate enough and make it easy to incorporate radiation and moisture effects as well as other substances in the flow. On the other hand, they bear a lot of empiricism and cannot deal with all kinds of complex flows. For the various length scale empirical relations that have been used, the reader can refer to [7]. Therry and Lacarrere [8] as well as Lascher and Arya [9] have done an important work in this field. The most common formulation used today for the length scale in the one-equation turbulent modelling is that of Duynkerke and Driedonks [10].

The most advanced models within the eddy viscosity concept are the two equation models. By introducing two transport equations for turbulent parametrization are theoretically flexible enough to treat any complex problem. A considerable amount of work exists in the open literature concerning this family of models especially in closed systems. The most widely used in this area are those utilizing the transport equations for the turbulent kinetic energy $\mathrm{k}$ and the turbulent energy dissipation $\varepsilon$ [11].

The eddy viscocity in this case is given by the relation [8]:

$$
K=c_{\mu} \frac{k^{2}}{\varepsilon}
$$

The so-called standard k- $\varepsilon$ model that has been extensively applied to neutral flows and it is based on the following transport equations for $\varepsilon$ [12],

$$
\frac{\partial \rho \varepsilon}{\partial t}+\frac{\partial \rho u_{j} \varepsilon}{\partial x_{j}}-\frac{\partial}{\partial x_{j}}\left(\rho \frac{K}{\sigma_{\varepsilon}} \frac{\partial \varepsilon}{\partial x_{j}}\right)=\rho S_{\varepsilon},
$$




$$
S_{\varepsilon}=C_{\varepsilon 1} \frac{\varepsilon}{k} S_{k}-C_{\varepsilon 2}^{\prime} \frac{\varepsilon^{2}}{k}
$$

with constant values of $c_{\mu}=0.09, \sigma_{\varepsilon}=1.3, C_{\varepsilon 1}=1.44$ and $C_{\varepsilon 2}^{\prime}=0.48$.

Concerning atmospheric flows, the standard $\mathrm{k}-\varepsilon$ model has been applied quite successfully for local scale neutral flow problems. However, in mesoscale flows where the Coriolis force is important, this model proved to be inappropriate without modifications. One major problem concerns is the over prediction of the boundary-layer depth and turbulent mixing [13]. On modelling the onedimensional atmospheric boundary layer, various modifications to the above equation have been proposed by several investigators (e.g. [14-17]).

Thus, it is clear that for the user that wants to use a two equation turbulence model with confidence in a large variety of atmospheric flows, it seems that there is not a common approach for both local and mesoscale level and there exist different views of what exactly to do in the mesoscale flow problems. In other words there is a 'modeling gap' between mesoscale and local scale whereas the ideal two equation model should be universal for both mesoscale and local scale flows and applicable to neutral and non neutral flows as well.

\section{A new approach in two equation turbulence modelling}

An attempt has been recently started by Bartzis [12] with the aim to develop a two equation model equally applicable to mesoscale and local scale flows taking into consideration not only the neutral but also the nonneutral flows as well. An important criterion was that such an approach needed to be as simple as possible but at the same time reliable and consistent with the experimental evidence and turbulence theory. The new approach is based on the following plausible assumptions:

a. The Prandtl's hypothesis $K \sim k^{\frac{1}{2}} \cdot \ell$ is valid

b. Any composite turbulent parameter $\xi$ is scaled as : $\quad \xi=k^{m} \cdot \zeta^{n} \quad \zeta=\frac{1}{\ell}$

c. The turbulent parameter $\xi$ (including $\mathrm{k}$ ) is subject to convection, diffusion and source i.e.

d. The $\mathrm{k}$ and $\xi$ parameters $(n \neq 0)$ are adequate in obtaining turbulence closure

e. The TKE equation is given by eq (2)

Based on the above assumptions, it can be proved [12] that the $\xi$-transport equation is given by the relation:

$$
\frac{\partial \rho \xi}{\partial t}+\frac{\partial \rho u_{j} \xi}{\partial x_{j}}-\frac{\partial}{\partial x_{j}}\left(\rho \frac{K}{\sigma_{k}} \frac{\partial \xi}{\partial x_{j}}\right)=\rho S_{\xi}
$$




$$
\begin{gathered}
S_{\xi}=m \frac{\xi}{k} S_{k}+n \frac{\xi}{\zeta} S_{\zeta}-2 m n \frac{K}{\sigma_{k}} \frac{\xi}{k \zeta} \frac{\partial \zeta}{\partial x_{j}} \cdot \frac{\partial k}{\partial x_{j}} \\
+m(1-m) \frac{K}{\sigma_{k}} \frac{\xi}{k^{2}}\left(\frac{\partial k}{\partial x_{j}}\right)^{2}+n(1-n) \frac{K}{\sigma_{k}} \frac{\xi}{\zeta^{2}}\left(\frac{\partial \zeta}{\partial x_{j}}\right)^{2}
\end{gathered}
$$

Eqn (5b) suggests as the $\xi$ parameter with simplest source term, the inverse length scale $\zeta$.

Its source term is suggested to be [12]:

$$
S_{\zeta}=-C_{\zeta} \rho k^{\frac{1}{2}}\left(\zeta-\frac{1}{\ell_{0}}\right)^{2}, C_{\zeta} \approx 1.0
$$

The parameter $\ell_{0}$ is an 'attractor characteristic length' imposed by the turbulence forcing and the flow global invariants. Such a length has been more or less proposed by Bartzis [5] for the atmospheric boundary layer:

$$
\frac{1}{\ell_{0}}=\frac{1}{C_{\Gamma}} \frac{f U_{g}}{k}+\frac{1}{C_{N}} \frac{N_{z}}{k^{\frac{1}{2}}}, C_{\Gamma} \approx 0.49, C_{\mathrm{N}} \approx 0.51
$$

where $U_{g}$ is the geostrophic wind, $\mathrm{f}$ is the Coriolis parameter and $N_{z}$ the Brunt-Vaisala frequency.

The present $\mathrm{k}-\zeta$ model is supposed to apply equally to local and mesoscale flows and also equally to neutral and non-neutral flows. The model has been up to now quite successful in predicting neutral atmospheric boundary layer characteristics and local flows over obstacles. The results were in all cases better than the standard k- $\varepsilon$ model [12]. In the present paper two additional applications are presented:

a. The O'Neil Nebraska atmospheric boundary layer growth experiment

b. The slanted roofs street canyon problem

The present turbulence model as well as the standard k- $\varepsilon$ model have been incorporated into the CFD local scale computer code ADREA ([1], [18]). It is a finite volume transient, three-dimensional, fully compressible transport code with emphasis on terrains of high complexity.

\section{The O'Neil Nebraska atmospheric boundary layer growth experiment}

The atmospheric boundary layer growth measured on 25 August 1953 at Great Planes, O'Neill, Nebraska during the morning hours has been calculated. The observed temperature profiles at various times are shown in Fig. 1a (Busch et al. [19]). 
The starting time for the calculations has been taken the 08:35 CST, i.e. when the first observed temperature data has been obtained. The ground heat flux profile used by Busch et al. [19] has been adopted, i.e.

$$
H(t)=0.25 \rho c_{p} \sin \left(2 n\left(t-t_{0} / 24 h\right) \quad t_{0}=06: 36 h\right.
$$

The calculation height is selected to be $2735 \mathrm{~m}$. This value has been generated by using totally 38 axial steps using the relation $\Delta z_{k} / \Delta z_{k-1}=1.1$

For the calculations a time step of $225 \mathrm{~s}$ has been selected on the ground that it gives good description of the ground heat flux curve. The predicted temperature profile is given in Fig. 1b. If one takes into consideration on one hand that the solar radiation is not explicated treated and on the other hand physical processes such as horizontal advection and synoptic vertical motions have been neglected, the results can be classified as quite satisfactory.
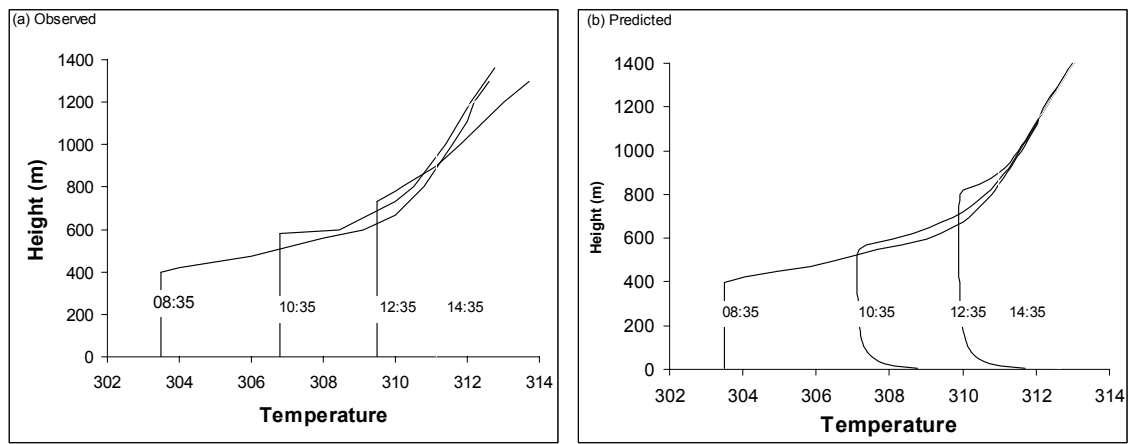

Figure 1: $\quad$ The O’Neill Nebraska Experiment inversion rise.

\section{The slanted roofs street canyon problem}

This application is related to the air pollution levels in urban areas. It refers to experimental measurements in an urban canyon configuration that have been performed in the Blasius wind tunnel of Hamburg University. It consisted of a two-dimensional span of identical parallel street canyons (Fig 2), normal to the oncoming wind and separated by buildings with slanted roofs. The canyon width to height ratio was equal to unity. The critical parameter under study in this family of problems is the concentration levels developed by emissions corresponding to cars passing through street canyons [20].

The modelling strategy has been selected from (Vlachogiannis et al. [21]). The wind tunnel input parameters have been scaled up to full scale. The computation domain comprised a 2-D grid of $130 \times 61$ cells generating 10 horizontal and 30 vertical grids within the canyon. The horizontal grid is uniform with $\Delta x=2.0 \mathrm{~m}$ covering totally six canyons. The vertical grid is uniform within the canyon and logarithmic above the canyon with $\Delta \mathrm{z}_{\min }=1 \mathrm{~m}$ and $\Delta \mathrm{z}_{\max }=6,5 \mathrm{~m}$, 
covering a domain five times the building height. The source was modelled as a continuous line source placed in the centre of the test canyon, ejecting the gas out horizontally at two opposite directions by imposing a horizontal obstacle of thickness $0.05 \mathrm{~m}$ and length $3.3 \mathrm{~m}$, at a distance of $0.4 \mathrm{~m}$ from the ground. The pollutant exit velocity was equal to $0.012 \mathrm{~ms}^{-1}$ in the $\mathrm{x}$-direction and the exit mass fraction was 0.01 . The pollutant exit vertical length has been taken equal to $0.35 \mathrm{~m}$ at each side. At the main inflow boundary of the 2-D simulation, the profile of the horizontal velocity was calculated by the model using the free stream velocity value of $U_{\infty}=5.85 \mathrm{~ms}^{-1}$, surface roughness length of $\mathrm{z}_{0}=0.6 \mathrm{~m}$ and displacement height above the ground of $\mathrm{D}=30 \mathrm{~m}$. The roughness for the street surface was estimated to correspond to the experiment at $0.1 \mathrm{~m}$ approximately.

The inlet flow has been generated by running the model as a 1-D problem with $\mathrm{U}_{\infty}=5.85 \mathrm{~ms}^{-1}$. The velocity, turbulent kinetic energy, the inverse length scale and the energy dissipation generated by the 1-D simulation, constitute the inlet boundary conditions for the 2-D problem.

To compare the model concentrations with experimental measurements, nondimensional values of the calculated concentrations were determined using the following relation:

$$
C^{*}=C U_{\delta} H L / Q
$$

where $C^{*}$ is the non-dimensional concentration, $C$ is the concentration, $U_{\infty}\left(\mathrm{ms}^{-1}\right)$ is the wind velocity measured in the free stream, $\mathrm{H}$ (in $\mathrm{m}$ ) is the height of building, Q (in $\mathrm{m}^{3} / \mathrm{s}$ ) is the source strength and L (in $\mathrm{m}$ ) the line source length.

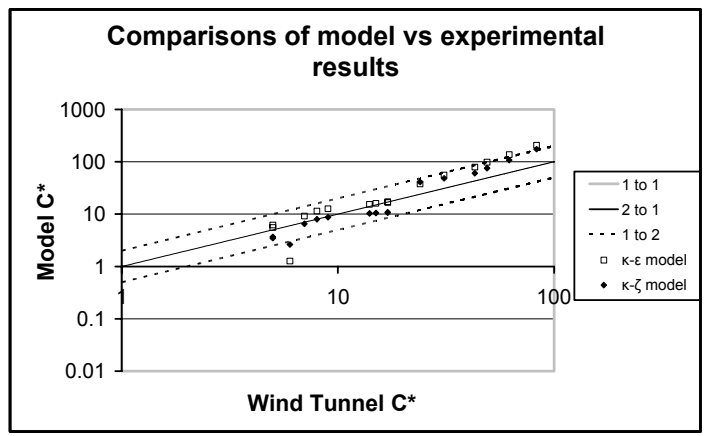

Figure 2: (a) Two-dimensional urban case of multiple parallel street canyons with slanted roofs and the locations of the source (in dark) and (b) the sensors positions in the test canyon.

Calculations have been performed with both the $\mathrm{k}-\zeta$ model and the standard $\mathrm{k}$ $\varepsilon$ model. Figure 3 shows the comparison between the calculated and measured concentrations at the sensor positions shown in Figure 4. It can be seen that for both models the agreement between calculated concentrations and measurements is rather good. The $\mathrm{k}-\zeta$ model shows a better agreement compared with the $\mathrm{k}-\varepsilon$ model especially in the area of high concentrations. 


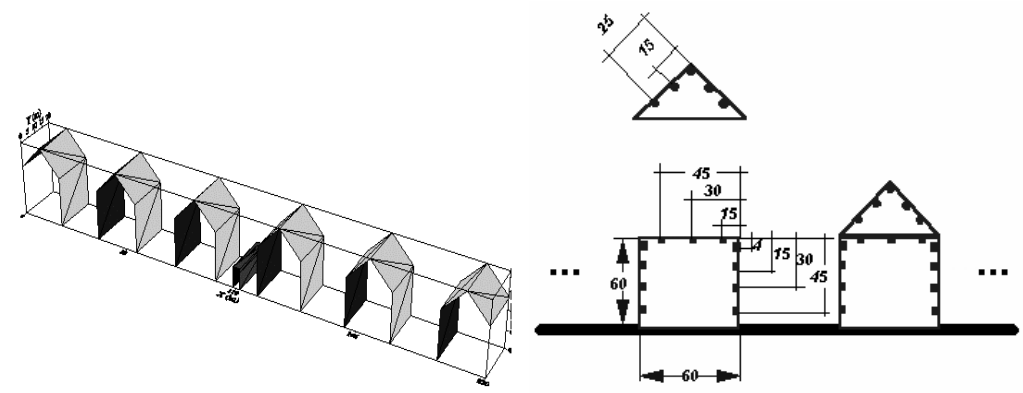

Figure 3: Comparison of non-dimensional concentration values of model against experimental results at 18 measurement positions (Fig. 2).

\section{Conclusions}

CFD-RANS methodology is the main tool used today for practical problems dealing with atmospheric boundary layer modelling and dispersion over terrains of high complexity.

The problem of turbulence closure scheme selection is still an open question in atmospheric flows.

For simple cases one-equation and simple empirical models can work satisfactorily

The two equation models seem to be appropriate to deal with practical complex problems

A real common approach in the area of two equation modeling in mesoscale does not exist. On the contrary, common approach in the area of two equation modeling in local scale exists (the standard k- $\varepsilon$ model)

There is a need to close the 'modeling gap' between mesoscale and local scale. The ideal two equation model should be universal for mesoscale and local scale

The new methodology can be considered as a first step towards developing a new general approach on ABL modelling, applied uniformly both on local scale and mesoscale, utilizing the two-equation modelling concept. An important criterion was that such an approach needed to be as simple as possible but at the same time reliable and consistent with the experimental evidence and turbulence theory.

A new methodology has lead among others, to:

- $\quad$ a transport equation for any turbulent parameter $\xi$

- an introduction of the synoptic properties of the flow in defining turbulence local characteristics

Further exploitation of this approach could lead to further improvements to a variety of the existing two equation models.

Selecting as $\xi$ parameter the inverse length scale $\zeta$, the obtained results are more than encouraging for the following reasons:

- The model can treat convective/neutral/stable atmospheric flows and it is relatively easy to be treated numerically due to its simplicity 
- The application to the O’Neill Nebraska Experiment shows satisfactory results on non neutral atmospheric boundary layer flows

- $\quad$ For the present urban scale application, the proposed $\mathrm{k}-\zeta$ model gave overall better results than the standard $\mathrm{k}-\varepsilon$ model

Can the abovementioned approach attract more applications enriched with benchmark exercises and intercomparison studies to lead to a standard two equation model applicable to both local and mesoscale flows?

\section{Acknowledgements}

The present work was conduced in the frame of COST 728. The author would like to thank Dr Th. Sfetsos and Mr G. Efthimiou for helping interpreting the present results as well as finalising the manuscript.

\section{References}

[1] Bartzis, J. Venetsanos, A., Varvayani, M., Catsaros, N., Megaritou, A., "ADREA-I: A three-dimensional transient transport code for complex terrain and other applications", Nuclear Technology, vol. 94, pp. 135-148, (1991).

[2] Mellor, G.L., Yamada, T., "A hierarchy of turbulence closure models for planetary boundary layers", Journal of the Atmospheric sciences, vol. 31, pp. 1791-1806, (1974).

[3] Mellor, G.L., Yamada, T., "Development of a turbulence closure model for geophysical fluid problems", Reviews of geophysics and space physics, vol. 20, issue 4, pp. 851-875, (1982).

[4] Prandtl, L., "Uber ein neues Formelsystem fur die ausgelbitete Turbulenz. In L. Prandtl Gesammete Abhandlungen, Springer-Verlag, Berlin, pp874 (1945).

[5] Bartzis, J.G., "Turbulent diffusion modelling for wind flow and dispersion analysis", Atmospheric Environment, vol. 23, issue 9, pp. 1963-1969, (1989).

[6] http://box.mmm.ucar.edu/mm5/ (documents mm5-desc* from: ftp://ftp.ucar.edu/mesouser/Documents).

[7] Holt, T., Raman, S., "A Review and Comparative Evaluation of Multilevel Boundary Layer Parameterisations for First Order and Turbulent Kinetic Energy Closure Schemes" Reviews of Geophysics and Space Physics, vol. 26, pp. 761-780, (1988).

[8] Therry, G., Lacarrère, P. "Improving the Eddy Kinetic Energy Model for the Planetary Boundary Layer Description", Boundary Layer Meteorology, vol. 25, pp. 63-88 (1983).

[9] Lacser A., and Arya, S.P.S. "A Comparative Assessment of Mixing Length Parameterizations in the Stably Stratified Nocturnal Boundary Layer", Boundary Layer Meteorology, vol. 36, pp. 53-70, (1986).

[10] Duynkerke, P.G., Driedonks, A.G.M. "A Model for the Turbulent Structure of the Stratocumulus-topped Atmospheric Boundary Layer", Journal of the Atmospheric sciences, vol. 44, pp. 43-64, (1987). 
[11] Launder, B. E., Reece, G. J., Rodi, W., " Progress in the Development of a Reynolds - Stress Turbulence Closure. J.” Fluid Mech., Vol.68, pp 313348. (1975).

[12] Bartzis, J. G.: "New approaches in the two-equation turbulence modeling for atmospheric applications" Boundary Layer Meteorology, Vol116, pp 445-459 (2005).

[13] Weng, W, Taylor, P.A.: 'On Modelling the One-Dimensional Atmospheric Boundary Layer', Boundary-Layer Meteorol. Vol107, pp 371-400. (2003).

[14] Detering, H. W., Etling, D., 'Application of the E - $\varepsilon$ Turbulence Model to the Atmospheric Boundary Layer', Boundary-Layer Meteorol. Vol 33, pp 113-133. (1985).

[15] Duynkereke, P. G., 'Application of the E - $\varepsilon$ Turbulence Model to the Atmospheric Boundary Layer', J. Atmos. Sci. Vol 45, pp 865-880, (1988).

[16] Andren, A., Moeng, C. H.: 'Single Point Closures in a Neutrally Stratified Boundary Layer', J. Atmos. Sci. Vol50, pp 3366-3379. (1993).

[17] Apsley, D. D., Castro, I. P., 'A Limited - Length Scale K - $\varepsilon$ Model for the Neutral and Stably - Stratified Atmospheric Boundary Layer', BoundaryLayer Meteorol. Vol 83, pp 75-98. (1997).

[18] Bartzis, J. G.,“ADREA-HF: A Three-Dimensional Finite Volume Code for Vapor Cloud Dispersion in Complex Terrain” JRC-ISPRA Report, EUR 13580, Italy. (1991).

[19] Busch N. E., Chang S. W. and Anthes R. A. "A multilevel model of the planetary boundary layer suitable to use with mesoscale dynamic models." J. appl. Met. Vol15, pp 909 (1976).

[20] Rafailidis, S., 'Influence of Building Areal Density and Roof Shape on the Wind Characteristics above a Town', Boundary-Layer Meteorol. Vol 85, pp 255-271. (1997).

[21] Vlachogiannis D., S. Rafailidis, J. G. Bartzis, S. Andronopoulos and A. G. Venetsanos, "Modelling of Flow and Pollution Dispersion in a Two Dimensional Urban Street Canyon", Water, Air and Soil Pollution-Focus, Vol 2, pp 405-417 (2002). 